\title{
Análisis de Estabilidad Transitoria en un Sistema Industrial con Generación Propia Interconectado con el Sistema de Potencia
}

\author{
Manuel F. Trebilcock, Francisco Santamaría, Jorge A. Alarcón \\ Universidad Distrital Francisco José de Caldas, Grupo de Compatibilidad e Interferencia Electromagnética, \\ GCEM, Crr 7 No 40B-53, piso 5, Bogotá D.C.-Colombia (e-mail: manfer901@yahoo.com, \\ fsantamariap@udistrital.edu.co, jaalarconv@udistrital.edu.co)
}

Recibido May. 14, 2013; Aceptado Jul. 23, 2013; Versión final recibida Sep. 16, 2013

\begin{abstract}
Resumen
Se presenta un estudio de estabilidad transitoria en un proceso industrial que incluye una planta de cogeneración de electricidad interconectada a una red central externa. Se evalúan problemas que se pueden presentar en este tipo de sistemas ante la desconexión de la red externa y se analiza la ocurrencia de fallas, como la pérdida de sincronismo y caída en frecuencia de la generación. También se discuten soluciones para una operación confiable y segura del sistema, entre los cuales se encuentran esquemas de deslastre de carga y el ajuste de protecciones. Este artículo muestra que los estudios de estabilidad transitoria permiten introducir acciones correctivas, definir criterios para establecer la prioridad de salida de las cargas y se puede observar el comportamiento del sistema ante cualquier problema. También es posible verificar los ajustes de las protecciones del sistema, garantizando la continuidad en el suministro de energía eléctrica en la planta industrial y su proceso productivo.
\end{abstract}

Palabras clave: cogeneración, electricidad, estabilidad, deslastre de carga, protecciones eléctricas

\section{Transient Stability Study in Industrial System Interconnected to the Power System}

\begin{abstract}
This article presents a transient stability study of an industrial process that includes an electricity cogeneration plant connected to an external central line. Problems that may occur due to disconnection from the external network are evaluated and faults such as loss of synchronism and frequency decline of the generation system are discussed. Also, possible solutions for a reliable and safety operation of the system, including load shedding and protections fitting, are discussed. This work shows that transient stability studies allow introducing corrective actions, defining criteria to establish priority of charges and observing the general behavior of the systems when problems occur. Also, it is possible to verify the corrective actions, guaranteeing the continuity of the electrical service in the plant and in its productive process.
\end{abstract}

Keywords: cogeneration, electricity, stability, load shedding, electrical protections 


\section{INTRODUCCIÓN}

En los últimos años, la industria eléctrica está pasando por un proceso de reestructuración en todo el mundo, con una clara tendencia de cambiar la producción de energía eléctrica desde grandes plantas de generación centralizadas hacia relativamente pequeños sistemas descentralizados. De hecho, la desregularización del mercado eléctrico, los requerimientos de continuidad de servicio, el interés de reducir las pérdidas y sobrecarga de los conductores en los sistemas de transmisión y distribución, el desarrollo de nuevas tecnologías y la preocupación por el medio ambiente, han dado paso a la interconexión de fuentes de generación de energía de mediana y baja potencia en diferentes puntos de la red eléctrica, desarrollando lo que se conoce actualmente como generación distribuida (Kheradmandi et al, 2011;Luna y Parra, 2011; Yang y Chen, 2005; Zayandehroodi et al, 2011).

Es importante tener en cuenta que, aunque el tipo más común de generación distribuida está relacionada con la generación asíncrona conectada a la red a través de inversores (Wang y Freitas, 2009), los generadores distribuidos sincrónicos son muy comunes (Granville et al, 2009; Mahat et al, 2009; Salim et al, 2011; Shinji et al, 2009); en particular en plantas industriales (Zayandehroodi et al, 2011), donde el uso de producción combinada de calor y electricidad (cogeneración) brinda mejoras substanciales en eficiencia y ahorro de energía, además de beneficios económicos (Nobile, 1987; Paine, 2002).

Las maquinas sincrónicas usadas para generación distribuida son más vulnerables a perder su estabilidad comparadas con los grandes generadores de la red interconectada, debido a los tiempos de despeje de falla relativamente altos y su baja inercia. Además, estos complejos industriales con autogeneración, al estar conectados a la red externa, no solo están expuestos a perturbaciones dentro de la planta, sino también a perturbaciones ocasionadas por eventos externos en el sistema interconectado (Bidram et al, 2012; Yang y Chen, 2005; Dunki-Jacobs y Davis, 1994; IEEE 399, 1997). Sin embargo, los estudios de estabilidad transitoria prácticamente no son requeridos en la etapa de diseño de sistemas industriales, y este proceso se basa principalmente en estudios como son flujos de carga, corto circuito, coordinación de protecciones y arco eléctrico (Dunki-Jacobs y Davis, 1994).

El propósito de los estudios de estabilidad transitoria es analizar el comportamiento de un sistema de potencia ante contingencias, perturbaciones y/o eventos transitorios y con base en estos estudios determinar las acciones que habrán de tomarse para permitir al sistema afrontar la ocurrencia de dichos eventos, y mantener su operación con las menores desviaciones posibles respecto de la condición de operación de pre-disturbio (en estado estable). Algunas de las acciones correctivas que pueden ser determinadas a partir de los estudios de estabilidad transitoria son: el tiempo crítico de liberación de falla para ajuste de protecciones y el diseño de esquemas para deslastre de carga (Zimmermann et al, 2000).

El propósito de los estudios de estabilidad transitoria es analizar el comportamiento de un sistema de potencia ante contingencias, perturbaciones y/o eventos transitorios y con base en estos determinar las acciones que habrán de tomarse para permitir al sistema afrontar su ocurrencia, manteniendo operación continua, con las menores desviaciones posibles respecto de la condición de operación de pre-disturbio (en estado estable). Algunas de las acciones correctivas que pueden ser determinadas a partir de los estudios de estabilidad transitoria son: el tiempo crítico de liberación de falla para ajuste de protecciones y el diseño de esquemas para deslastre de carga (Zimmermann et al, 2000).

Hoan y Chow (1986), discuten el diseño de una planta de emergencia en un complejo industrial, revisando problemas de estabilidad como sobrecargas, caídas de voltaje y frecuencia, que pueden causar la pérdida del suministro de energía eléctrica del complejo en condiciones de emergencia, determinando esquemas de deslastre y arranque de cargas para evitar problemas de estabilidad en operaciones de contingencia en el sistema analizado. Dunki-Jacobs y Davis (1994), muestran la importancia de los estudios de estabilidad en complejos industriales mediante un ejemplo real, en el cual, debido a la mala planificación del sistema, fue necesario realizar un rediseño del mismo, debido a que las corrientes aportadas por los generadores instalados en la planta hacían indispensable el uso de limitadores de corriente y estos no permitían aprovechar el $100 \%$ de la capacidad de potencia de los generadores.

Zimmermann et al (2000), llevaron a cabo un estudio de estabilidad transitoria en un parque industrial, proponiendo un esquema de deslastre de carga por bajo voltaje, restringido por la exportación de corriente hacia la red externa. Además, evalúan los tiempos críticos para el despeje de fallas externas, para así establecer los retardos de cada escalón del esquema de deslastre de carga por bajo voltaje y evitar la operación de las protecciones por eventos en la red externa.

Salim et al (2011), concluyen que los problemas de estabilidad en sistemas de distribución con generadores sincrónicos distribuidos, si no son resueltos, pueden traer otros inconvenientes al sistema. En especial las 
oscilaciones electromecánicas, las cuales causan problemas relacionados con la calidad de potencia. En el caso de los complejos industriales, donde la generación se encuentra cerca de los centros de consumo; las variaciones tanto en frecuencia como en voltaje generadas por dichas oscilaciones, pueden afectar cargas sensibles. Bidram et al (2012), muestran a partir de simulaciones en un complejo industrial con dos centros de generación interconectados con la red externa, como con restricciones de carga para generación distribuida con máquinas sincrónicas, se puede asegurar la estabilidad de su primera oscilación después de una falla.

Este artículo presenta un estudio que permite observar la importancia de los análisis de estabilidad en sistemas industriales con autogeneración basada en máquinas sincrónicas e interconectados a la red, mediante la simulación de un sistema de potencia industrial en un software de análisis de sistemas de potencia, mostrando inconvenientes comunes que se pueden presentar en este tipo de sistemas y su impacto en el mismo, brindando posibles soluciones a los problemas encontrados y discutiendo aspectos que se deben tener en cuenta al momento de analizar el sistema.

Uno de los mayores retos al momento de realizar este tipo de análisis es contar con toda la información del sistema, a partir de la cual es posible obtener resultados confiables de las simulaciones. En este artículo se presentan los datos de entrada y estrategias empleadas para obtener la información que no se puede adquirir de manera directa, garantizando la utilidad y confiabilidad de los resultados obtenidos. La organización de este artículo es la siguiente: inicialmente se presenta el sistema eléctrico de la planta industrial bajo análisis. Luego se muestran los resultados de las simulaciones de los diferentes casos de estudio. Posteriormente se presenta una sección de discusión en la que se presentan soluciones a los problemas encontrados a partir de las simulaciones; y finalmente se presentan las conclusiones, en donde se resalta la importancia de la realización estudios de estabilidad en plantas industriales con autogeneración.

\section{METODOLOGÍA}

El objetivo de este artículo es resaltar la importancia de los estudios de estabilidad transitoria en complejos industriales con autogeneración e interconectados a la red. Para cumplir tal propósito se modeló el sistema eléctrico de una planta industrial usando una herramienta computacional para análisis de sistemas de potencia. Una vez implementado el modelo, se simularon diferentes casos de estudio, que permitieron analizar el comportamiento de la planta de cogeneración del complejo industrial ante la ocurrencia de fallas en la red externa y la pérdida de la interconexión con el sistema de potencia. Finalmente, a partir de los resultados se propusieron soluciones o medidas de control que permiten minimizar o eliminar los efectos negativos en la estabilidad del sistema eléctrico debido a la incorporación de generación distribuida.

\section{Características y Configuración de la Red}

Para desarrollar este trabajo se empleó el esquema eléctrico simplificado de una planta industrial, el cual se presenta en la Fig. 1. La interconexión del complejo industrial con la red eléctrica se realiza mediante una línea de $44 \mathrm{kV}$ de dos circuitos (L1 y L2) a través de dos transformadores (T1 y T2) de 16 MVA (refrigeración forzada) de capacidad y relación de transformación 44/6.3 kV, de los cuales uno es de respaldo en caso de que una de las líneas o transformadores estén fuera de servicio por falla o mantenimiento.

La carga total instalada en la planta es de 14.4 MVA y la mayor parte de ella se encuentra conectada en niveles de tensión de $6.3 \mathrm{kV}$, donde se encuentran instalados los motores con potencias más grandes. La planta de cogeneración está conectada en la barra SWG_N2 del complejo industrial, la cual consta de un generador de 10 MVA. Los datos de elementos como motores, transformadores y cables fueron tomados de la recolección de información hecha en la planta industrial, y para modelar la planta de cogeneración fueron usados valores típicos reportados en la literatura.

Para modelar las constantes de la máquina sincrónica fue usada la tabla de valores promedio típicos de sus constantes, tomada de (Glover y Sarma, 2002). Para el valor la constante de inercia $\mathrm{H}$, fue usada la figura de energía almacenada en turbogeneradores de vapor, turbina incluida, tomada de (Kimbark, 1995). Aunque el modelado del generador requiere como mínimo lo anteriormente presentado para llevar a cabo un estudio de estabilidad, cada máquina también debe estar representada por un modelo del sistema de excitación y gobernación para obtener resultados más exactos, y en algunos casos garantizar que la unidad es estable en el régimen transitorio. Los modelos de reguladores de velocidad y turbinas son necesarios para evaluar la estabilidad de largo y corto plazo en respuesta a las oscilaciones de la frecuencia del generador (IEEE 399, 1997). Para el sistema de excitación se usó el modelo ST1, el cual representa todos los sistemas en los cuales la fuente de excitación es suministrada a través de un transformador desde los 
terminales del generador y es regulado por medio de un rectificador controlado (IEEE Std 421.5, 2005). Para el modelado del regulador de voltaje se usó el modelo TGOV1, el cual es un modelo simplificado para turbinas de vapor.

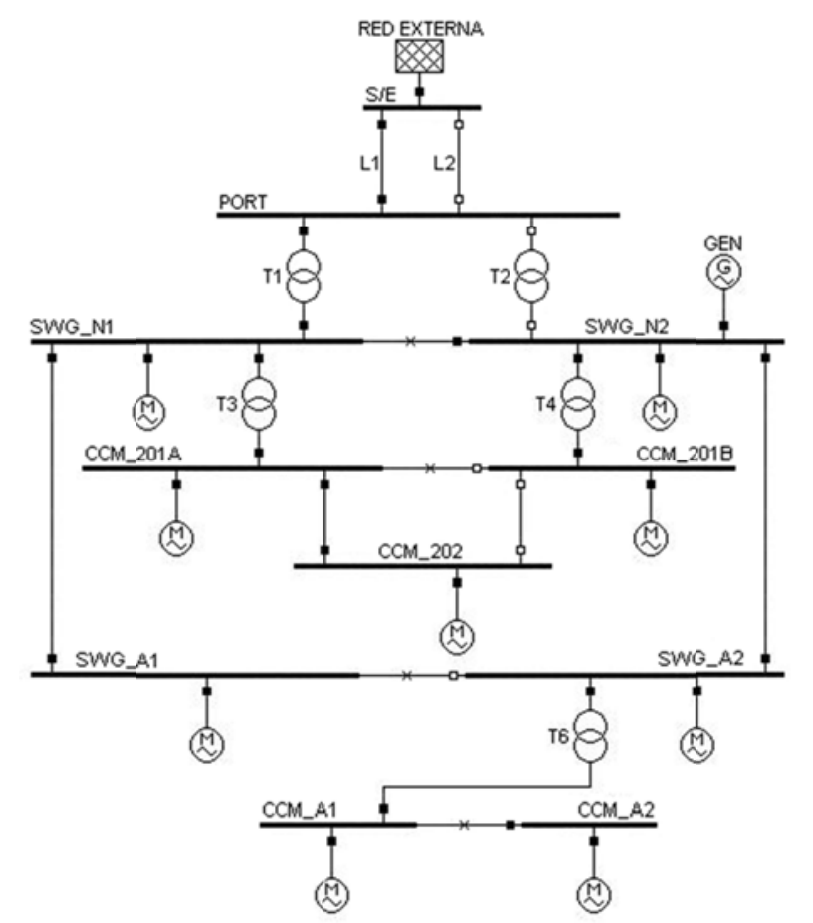

Fig. 1: Diagrama unifilar general de la planta industrial

En este caso se simuló la planta de generación como un nodo PQ, operando a su capacidad nominal, es decir 10 MVA, con un factor de potencia constante. Estos parámetros de simulación se eligieron debido a que el operador de red normalmente requiere que la generación distribuida opere con un factor de potencia constante, ya que esta condición permite la operación de la generación pero también limita el impacto de ésta en los perfiles de voltaje en el sistema de potencia (IEEE 1547, 2008).

\section{SIMULACIONES Y RESULTADOS}

\section{Comportamiento de la Planta de Cogeneración ante Fallas Externas}

Generalmente la inercia de las unidades de cogeneración es más pequeña que la de los generadores de la red, por lo que éstas tienden a responder más rápido ante perturbaciones en el sistema. Si la falla ocurre en la proximidad de la planta industrial y no es despejada antes de un determinado tiempo, la velocidad de la unidad de cogeneración dentro de la planta se podría incrementar rápidamente y ésta unidad podría perder el sincronismo (IEEE 399, 1997).

El tipo y la localización de la falla, así como el tiempo de despeje de ésta, son factores que afectan la estabilidad de la unidad de generación. Entre mayor la duración de una perturbación en el sistema, mayor la desviación de la frecuencia y del ángulo del rotor de la máquina sincrónica. Así, la duración de la falla afecta la capacidad de recuperación del sistema después de una perturbación severa (IEEE 399, 1997).

En este estudio, la estabilidad transitoria es analizada para fallas trifásicas, ya que generalmente éste tipo de falla es el más severo, es decir que si el sistema permanece estable para éste tipo de falla, permanecerá igualmente estable para otras fallas menos severas (Kimbark, 1995). De otro lado, la inestabilidad de un sistema eléctrico de potencia puede manifestarse de diferentes formas, una de ellas involucra las oscilaciones electromecánicas inherentes al sistema. Es por esto que la forma en que varía el ángulo de los rotores de los generadores ante perturbaciones es fundamental (Calderón-Guizar, 2010).

En los estudios de estabilidad transitoria el periodo de interés está delimitado de 3 a 5 s después de ocurrida la falla, sin embargo este periodo se podría extender hasta $10 \mathrm{~s}$ para sistemas grandes; si se determina que las máquinas del sistema permanecen en sincronismo dentro de este periodo de tiempo se considera que el sistema es estable (Grainger y Stevenson, 1996). En la práctica, ingenieros familiarizados con estudios de estabilidad esperan que las oscilaciones de los rotores de las máquinas se amortigüen hasta un nivel aceptable dentro de los 6 s después de una perturbación (IEEE 399, 1997). 
Durante la ocurrencia de una perturbación el ángulo del rotor del generador oscila. En un evento estable estas oscilaciones son amortiguadas y eventualmente desaparecerán hasta que el ángulo del rotor regrese a su valor de estado estable, como se muestra en la Fig. 2. Por otro lado, para una condición inestable el ángulo del rotor del generador oscila sin control, como se muestra en la Fig. 3.

Para observar los efectos de la fallas en la autogeneración de una planta industrial, se simuló una falla trifásica en la línea L1 (circuito de alimentación externa), seguido del despeje de la falla por medio de la apertura de los interruptores asociados a la línea, y finalmente el recierre de la línea. Adicionalmente, para observar el impacto de la localización de la falla en el generador del complejo industrial, se varió la distancia de la falla en la línea L1 tomando distancias de $0.43 \mathrm{~km}, 2.15 \mathrm{~km}$ y $3.7 \mathrm{~km}$.

La Fig. 2 muestra los resultados de las simulaciones y evidencia el impacto que la localización de la falla tiene en el ángulo del rotor del generador. Como se observa en esta figura, entre más cercana la falla a la planta de cogeneración, será mayor la variación inicial del ángulo del rotor del generador y más severas las oscilaciones, aunque también se amortiguan más rápidamente dando como resultado unos tiempos de estabilización similares en los tres casos.

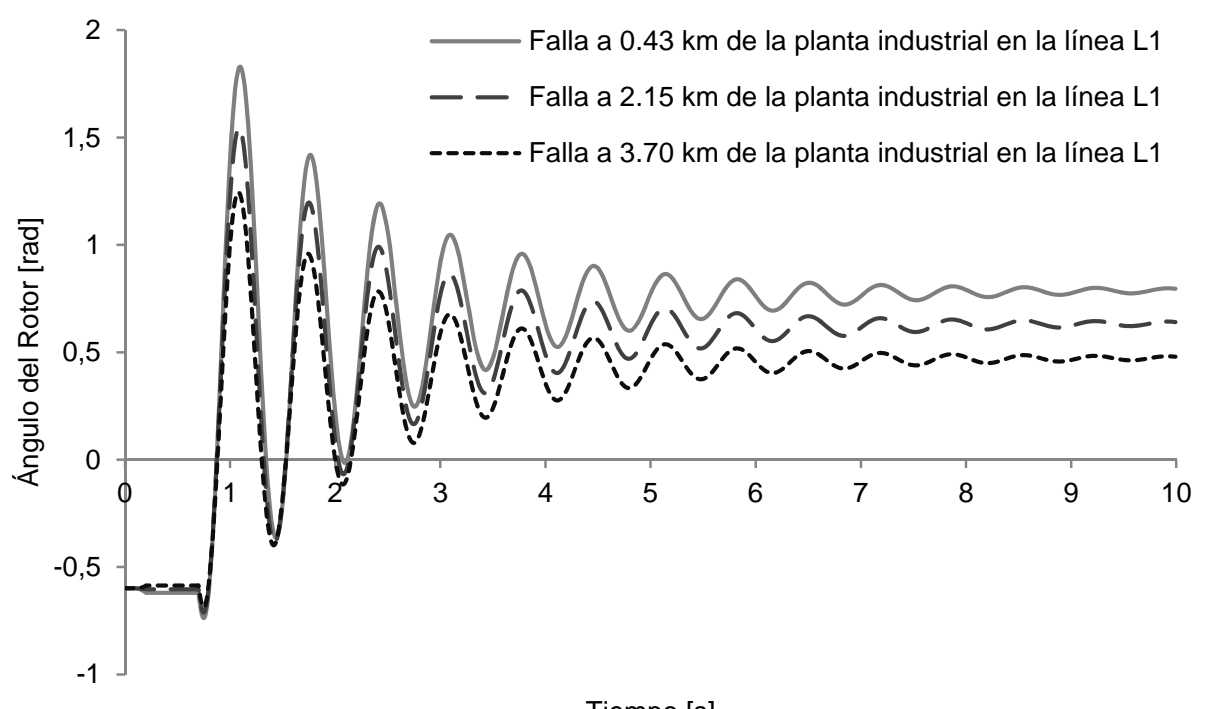

Tiempo $[\mathrm{s}]$

Fig. 2: Impacto de la localización de la falla en el ángulo del rotor de la generador

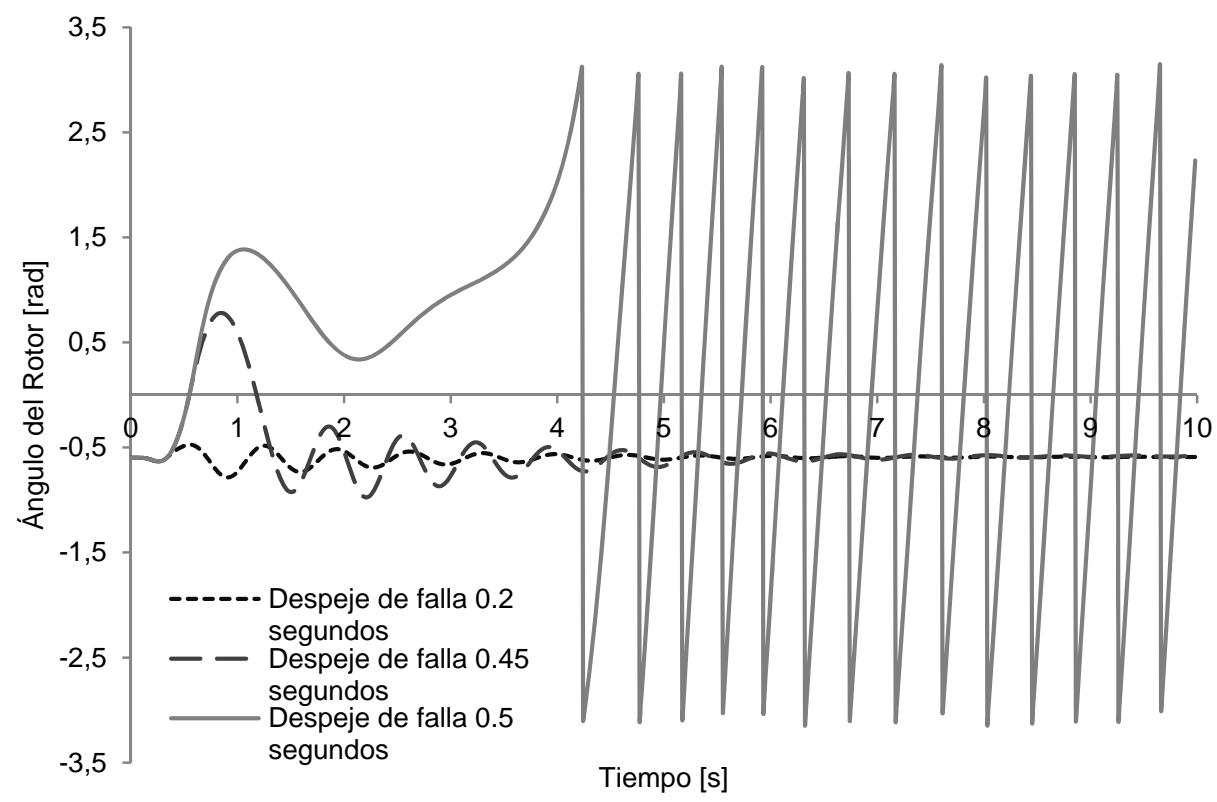

Fig. 3: Impacto del tiempo de liberación de la falla en el ángulo del rotor 
De otro lado, para evaluar el impacto del tiempo de despeje de la falla en la estabilidad del sistema, se varió el tiempo de despeje en una falla ocurrida a $0.43 \mathrm{~km}$ de la planta industrial en la línea L1. Como se observa en la Fig. 3, el generador pierde el sincronismo en un tiempo de liberación de falla de $0.5 \mathrm{~s}$, por lo tanto se puede concluir que un tiempo crítico para la liberación de esta falla es de aproximadamente 0.45 s.

\section{Operación Aislada del Complejo Industrial}

Cuando ocurre una perturbación en un complejo industrial con autogeneración interconectado con el sistema de potencia, y la interconexión entre la planta industrial y la red externa se pierde, el sistema industrial queda operando de manera aislada. Esto no es un problema si hay un balance entre la carga y la generación en la planta industrial. Sin embargo, cuando la carga excede la generación, hay problemas de estabilidad que podrían conllevar a una pérdida total del suministro de energía eléctrica (IEEE 399, 1997). En un sistema eléctrico de potencia, la frecuencia es una medida del balance generación - carga. Este balance, en una planta industrial que opera aislada, depende de varios factores como lo son la disponibilidad del combustible para la turbina del generador y el perfil de carga de la industria, que en muchos casos es variable debido a que depende del proceso productivo.

Con el propósito de evaluar el impacto que diferentes condiciones de sobrecarga tienen en la frecuencia del generador, se varió el factor de demanda de las cargas de la planta industrial a un 80,90 y $100 \%$, para variar el nivel de potencia suministrada por la red externa, posteriormente, se simuló la desconexión de la red externa después de $0.1 \mathrm{~s}$ iniciada la iteración ( $0.1 \mathrm{~s}$ después de ocurrida la falla), mediante la apertura de los interruptores asociados a la línea L1.

En los casos simulados, debido a que el complejo industrial estaba consumiendo potencia de la red externa antes de la desconexión, la frecuencia de la planta de cogeneración va a disminuir. Como se observa en la Fig. 4, entre mayor es la sobrecarga mayor es el cambio en la frecuencia y menor su valor de estabilización. Esta es la razón por la cual es necesario un esquema de deslastre de carga por baja frecuencia para garantizar la continuidad del servicio de las cargas críticas para el proceso productivo (IEEE 399, 1997). De manera similar, si la planta estuviera exportando energía al sistema de potencia, después de que se produce la desconexión, la frecuencia dentro de la planta industrial aumentaría según el exceso de potencia en la generación, aunque eventualmente regresaría a su valor nominal dependiendo de la respuesta del gobernador de la máquina.

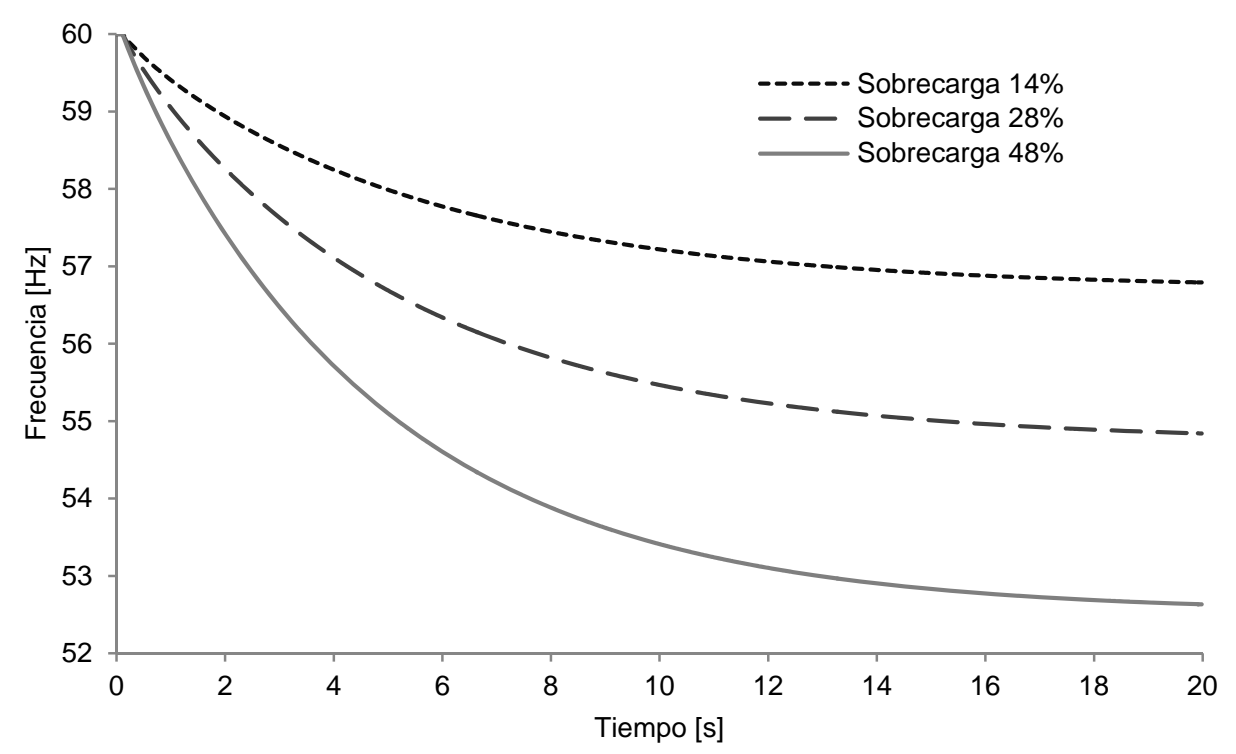

Fig. 4: Comportamiento de la frecuencia del generador ante sobrecargas generadas por la desconexión de la red externa

\section{DISCUSIÓN}

Como se observa en las figuras 2 y 3, las fallas generan oscilaciones en el ángulo del rotor de la máquina sincrónica, dichas oscilaciones también se ven reflejadas en las señales de voltaje y frecuencia del generador, las cuales pueden tener una duración o magnitud suficientes para afectar el desempeño de la industria, debido a la alteración de cargas sensibles como lo son los variadores de frecuencia y PLCs comunes en plantas industriales. Además, pueden ocasionar disparos innecesarios en protecciones, razón 
por la cual es recomendable usar los resultados de los estudios de estabilidad para establecer los tiempos de ajuste de las protecciones del sistema, con el fin de mejorar la confiabilidad del sistema y la eficacia de los esquemas de protección propuestos

Al momento de ajustar las protecciones es necesario tener en cuenta el tiempo total de despeje de la falla, para que ante una falla el generador no pierda la estabilidad; este tiempo incluye los tiempos de operación según la curva característica del dispositivo de protección y el tiempo de operación del interruptor, además de un margen de seguridad. En este caso como se concluyó en la sección anterior, el tiempo crítico para la liberación de esta falla es de aproximadamente $0.45 \mathrm{~s}$, por lo cual la protección debería ser ajustada en 0.35 $\mathrm{s}$, lo cual deja un margen de seguridad de $0.1 \mathrm{~s}$.

La pérdida de bloques de alimentación ocasiona disminución en la frecuencia y el voltaje del sistema, como se puede observar en la Fig. 4. En este caso se muestra el efecto de las sobrecargas en la frecuencia del generador a causa de la pérdida de la alimentación de la red externa. Estas frecuencias, que se pueden presentar en casos de contingencia, son perjudiciales para el conjunto turbogenerador, y hay que tenerlas en cuenta para el esquema de deslastre de carga. Además de lo mencionado anteriormente, se deben tener en cuenta otros aspectos al momento de definir el esquema de deslastre de carga, como lo son:: la importancia de la carga a deslastrar para el proceso productivo, el número de escalones y la cantidad de carga a deslastrar, el tiempo de operación de las protecciones para evitar que la frecuencia caiga hasta zonas peligrosas, y el ajuste de los retardos en los sistemas de protección para prevenir disparos indeseados por eventos transitorios, tales como oscilaciones de potencia, arranque de motores y fallas externas. Esto indica que debe existir una adecuada coordinación con el esquema de protección.

Generalmente, en las plantas industriales se emplean relés en todas las celdas de media tensión. En la actualidad se cuenta con dispositivos multifuncionales los cuales tienen varias funciones de protección (IEEE 242, 2001). Por tal caso, en cada alimentador que posea relé y tenga función $81 U$ (baja frecuencia) se debe ajustar el disparo de la función $81 \mathrm{U}$ del alimentador, si éste hace parte de las cargas a deslastrar, de acuerdo con el esquema de deslastre de carga por frecuencia establecido para la planta. Este tipo de relés, en conjunto con los sistemas de monitoreo y supervisión remota permiten controlar, supervisar y administrar la generación, distribución y suministro de energía eléctrica de la planta industrial.

\section{CONCLUSIONES}

A través del análisis llevado a cabo y los resultados obtenidos en este trabajo, es posible concluir que en plantas industriales donde existe o está planeado instalar generadores distribuidos sincrónicos, la estabilidad es un aspecto muy importante a tener en cuenta. Estos sistemas, pueden presentar problemas como la caída en frecuencia debido a la desconexión de la red externa y oscilaciones que se presentan en el generador debido a una falla, que pueden conllevar a la pérdida de sincronismo de la máquina dependiendo de su cercanía y tiempo de liberación.

A partir de estudios de estabilidad, se pueden establecer criterios de diseño y determinar acciones correctivas, como modificaciones al tiempo crítico de despeje de fallas para el ajuste de las protecciones. Igualmente, se pueden definir criterios para establecer la prioridad de salida de las cargas en las etapas de deslastre de carga de acuerdo con su relevancia en los procesos industriales, y se puede observar el comportamiento del sistema ante contingencias y fallas. También es posible verificar los ajustes de las protecciones del sistema, para demostrar la confiabilidad de los esquemas propuestos, garantizando la continuidad en el suministro de energía eléctrica en la planta industrial y su proceso productivo.

\section{REFERENCIAS}

Bidram, A., M. E. Hamedani-golshan y A. Davoudi, Loading constraints for first swing stability margin enhancement of distributed generation, IET Generation, Transm. \& Distribution, 6(12), 1292 - 1300 (2012).

Calderón-Guizar, J. G., Estudios de estabilidad transitoria en sistemas eléctricos industriales con generación propia interconectados con el sistema de transmisión, Ingeniería Investigación y Tecnología, XI (4), 445-451 (2010).

Dunki-Jacobs, J. R. y C. E. Davis, An Argument and Procedure for Conceptual Power System Design Studies, IEEE Transactions on Industry Applications, 30 (2), 253-261 (1994).

Glover J. D. y M. S. Sarma, Sistemas de Potencia Análisis y Diseño, $3^{a}$ edición, Thompson Learning, México D. F., México (2002). 
Grainger, J. J. y W. D. Stevenson, Análisis de Sistemas de Potencia, México D. F., México, Mc Graw-Hill, (1996).

Granville, S., P. Lino, F. Ralston, L. Barroso y M. Pereira, Recent advances of sugarcane biomass cogeneration in Brazil, IEEE Power and Energy Society General Meeting, 1-5, Calgary, Canada, 26 a 30 de Julio (2009).

Hoan, W. T., y M. Chow, Transient Stability Analysis for Sohio Prudhoe Bay Emergency Power System, IEEE Transactions on Industry Applications: 22(3), 430-434 (1986).

IEEE 399 (Brown Book): IEEE Recommended Practice for Industrial and Commercial Power Systems Analysis (1997).

IEEE 242 (Buff Book): IEEE Recommended Practice for Protection and Coordination of Industrial and Commercial Power Systems (2001).

IEEE Std 421.5: IEEE Recommended Practice for Excitation System Models for Power Stability Studies, (2005).

IEEE Std 1547: IEEE Standard for Interconnecting Distributed Resources with Electric Power Systems (2008).

Kheradmandi, M., R. Feuillet, M. Ehsan y N. Hadj-Saied, Allocating the cost of transient stability constraint relief in bilateral electricity markets, IET Generation, Transmission \& Distribution, 5 (11), 1124-1131 (2011).

Kimbark, E. W., Power System Stability, Volume I: Elements of Stability Calculations, USA: IEEE Press (1995).

Klein, M., C. J. Rogers y P. Kundur, a fundamental study of inter-area oscillations in power systems, IEEE Transactions on Power Systems, 6 (3), 914 - 921 (1991).

Luna, L. y E. Parra, Methodology for assessing the impacts of distributed generation interconnection, Revista Ingeniería e Investigación, 31 (2), 36-44 (2011).

Mahat, P., Z. Chen y B. Bak-Jensen, Gas turbine control for islanding operation of distribution systems, IEEE Power and Energy Society General Meeting, 1-7, Calgary, Canada, 26 a 30 de Julio (2009).

Nobile, P. A., Power System Studies for Cogeneration: What's Really Needed?, IEEE Transactions on Industry Applications, 23 (5), 777-785 (1987).

Paine, D. M., Increasing the Electrical Output of a Cogeneration Plant, IEEE Transactions on Industry Applications, 38 (3), 726-735 (2002).

Salim, R. H., M. Oleskovicz y R. Ramos, Power quality of distributed generation systems as affected by electromechanical oscillations - definitions and possible solutions, IET Generation, Transmission \& Distribution, 5 (11), 1114 - 1123 (2011).

Shinji, T., A. Yokoyama e Y. Hayashi, Distributed generation in Japan, IEEE Power and Energy Society General Meeting, 1-5, Calgary, Canada, 26 a 30 de Julio (2009).

Wang, X. y W. Freitas, Influence of voltage positive feedback anti-islanding scheme on inverter-based distributed generator stability, IEEE Transactions on Power Delivery, 24 (2), 972-973 (2009).

Yang, W. C. y T. H. Chen, Analysis of Interconnection Operation of a Radial Feeder with a Cogeneration Plant, Power Engineering Society General Meet., 2384-2389, San Francisco, USA, 12 a 16 de Junio (2005).

Zayandehroodi, H., A. Mohamed, H. Shareef y M. Mohammadjafari, Impact of distributed generations on power system protection performance, International Journal of the Physical Sciences, 6 (16), 3873-3881 (2011).

Zimmermann, W. S., S. Hopp, M. Bondeur y D.-N. Chen, Transient stability study of the Hsin Yu CoGeneration Plant in Hsin-Chu Science Based Industrial Park in Taiwan, Power Engineering Society Winter Meeting, 2384 - 2389, Singapur, Singapur, 23 a 27 de Enero (2000). 